\title{
Regulatory mechanism of human vascular smooth muscle cell phenotypic transformation induced by NELIN
}

\author{
CHANGAN PEI ${ }^{1}$, SHIYONG QIN ${ }^{2}$, MINGHAI WANG ${ }^{2}$ and SHUGUANG ZHANG ${ }^{2}$ \\ ${ }^{1}$ Department of Vascular Surgery, Weifang People's Hospital, Weifang, Shandong 261041; ${ }^{2}$ Department of Vascular Surgery, \\ Qianfoshan Hospital Affiliated to Shandong University, Jinan, Shandong 250014, P.R. China
}

Received June 6, 2014; Accepted February 19, 2015

DOI: $10.3892 / \mathrm{mmr} .2015 .4365$

\begin{abstract}
Vascular disorders, including hypertension, atherosclerosis and restenosis, arise from dysregulation of vascular smooth muscle cell (VSMC) differentiation, which can be controlled by regulatory factors. The present study investigated the regulatory mechanism of the phenotypic transformation of human VSMCs by NELIN in order to evaluate its potential as a preventive and therapeutic of vascular disorders. An in vitro model of NELIN-overexpressing VSMCs was prepared by transfection with a lentiviral (LV) vector (NELIN-VSMCs) and NELIN was slienced using an a lentiviral vector with small interfering (si)RNA in another group (LV-NELIN-siRNA-VSMCs). The effects of NELIN overexpression or knockdown on the phenotypic transformation of human VSMCs were observed, and its regulatory mechanism was studied. Compared with the control group, cells in the NELIN-VSMCs group presented a contractile phenotype with a significant increase of NELIN mRNA, NELIN protein, smooth muscle (SM) $\alpha$-actin and total Ras homolog gene family member A (RhoA) protein expression. The intra-nuclear translocation of $\mathrm{SM} \alpha$-actin-serum response factor (SM $\alpha$-actin-SRF) occurred in these cells simultaneously. Following exposure to Rho kinsase inhibitor Y-27632, SRF and $\mathrm{SM} \alpha$-actin expression decreased. However, cells in the LV-NELIN-siRNA-VSMCs group presented a synthetic phenotype, and the expression of NELIN mRNA, NELIN protein, $\mathrm{SM} \alpha$-actin protein and total RhoA protein was decreased. The occurrence of SRF extra-nuclear translocation was observed. In conclusion, the present study suggested that NELIN was able to activate regulatory factors of SM $\alpha$-actin, RhoA and SRF successively in human VSMCs cultured in vitro. Furthermore, NELIN-induced phenotypic transformation of human VSMCs was regulated via the RhoA/SRF signaling pathway. The results
\end{abstract}

Correspondence to: Dr Shuguang Zhang, Department of Vascular Surgery, Qianfoshan Hospital Affiliated to Shandong University, 16766 Jingshi Road, Jinan, Shandong 250014, P.R. China

E-mail: shuguang_zhang@yeah.net

Key words: NELIN, human vascular smooth muscle cell, Ras homolog gene family member A/serum response factor signaling pathway, phenotype transformation of the present study provide a foundation for the use of NELIN in preventive and therapeutic treatment of vascular remodeling diseases, including varicosity and atherosclerosis.

\section{Introduction}

Vascular smooth muscle cells (VSMCs) are highly differentiated cells maintaining phenotypic plasticity. Dysregulation of phenotype switching from contractile phenotype to a synthetic phenotype is associated with vascular disorders, including hypertension, atherosclerosis and restenosis (1-4). Thus, VSMC differentiation is an essential component of vascular development.

NELIN, a gene expressed in cardiac muscles, was cloned on the basis of heart cDNA library screening in 1999 (5). NELIN protein was shown to be an actin-binding protein (ABP) by Wang et al (6). According to a previous study by our group (7), NELIN functions as a regulatory factor in maintaining the contractile phenotype of VSMCS and regulating the phenotypic transformation of VSMCs. However, the mechanism underlying the regulation of the phenotypic transformation of VSMCs by NELIN has remained elusive.

Ras homolog gene family member A (RhoA) belongs to the Rho family of small guanine triphosphatases (8) and activates the promotion of F-actin stress fiber formation and focal adhesion, which link stress fibers to the plasma membrane. Therefore, it affects the contractility and adhesion of VSMCs (9). RhoA regulates numerous cellular functions, including excessive contraction, phenotypic transformation, migration and proliferation (10), whose dysregulation is implicated in cardiovascular disorders, including hypertension, atherosclerosis and restenosis (11). It has been reported that RhoA/the Rho kinase signaling pathway participates in the vascular remodeling process and affects VSMC differentiation by regulating nuclear translocation of serum response factor (SRF) as well as actin polymerization (12).

In the present study, NELIN-overexpressing or -silenced VSMCs were established by infection with lentiviral vectors (LVs). Smooth muscle (SM) $\alpha$-actin was used as a marker protein of different VSMC phenotypes. The association between NELIN expression and VSMC differentiation was observed and the effect of NELIN expression levels on VSMCs differentiation was analyzed. Furthermore, the involvement of the RhoA/SRF signaling pathway in this process was 
Table I. Primer sequences for genes.

\begin{tabular}{lll}
\hline Gene & Forward primer $\left(5^{\prime}-3^{\prime}\right)$ & Reverse primer (5'-3') \\
\hline NELIN & TCCACGCGGAAAGAAGTACC & GCAAGCATTTCTTTAATCTCAGCC \\
$\alpha$-smoth muscle-actin & AGCTTTCAGCTTCCCTGAACA & AGAGCCATTGTCACACACCA \\
GAPDH & TGTTCGTCATGGGTGTGAAC & ATGGCATGGACTGTGGTCAT \\
\hline
\end{tabular}

discussed. The present study provided data which may serve as a foundation for the use of NELIN in the prevention and treatment of vascular remodeling disorders.

\section{Materials and methods}

Materials. Fetal bovine serum (FBS), Dulbecco's modified Eagle's medium (DMEM) and pancreatic enzymes were obtained from Gibco-BRL (Invitrogen Life Technologies, Carlsbad, CA, USA). TRIzol was purchased from Invitrogen Life Technologies. RNAi-Mate transfection reagent, interfering (NELIN siRNA forward, 5'-GCCCUGGUAAACUCAAACUTT-3' and reverse, 5'-AGU UUGAGUUUACCAGGGCTT-3')/overexpression lentivirus, shuttle plasmid and packaging plasmid (pGag/Pol, pRev, pVSV-G) were manufactured by Shanghai Gene Pharma Co. Ltd. (Shanghai, China). Puromycin, paraformaldehyde, TritonX-100, CBB, xylene, neutral balsam and glycerol were purchased from Shanghai Haoran Biological Technology Co., Ltd. (Shanghai, China). PrimeScript ${ }^{\circledR} 1$ st Strand cDNA Synthesis kit and SYBR ${ }^{\circledR}$ Premix Ex Taq kit were purchased from Takara Bio Inc. (Otsu, Japan). SDS, $\beta$-mercaptoethanol, bromophenol blue and Tris-buffered saline/Tween (TBST) were purchased from Shanghai Bogu Biotech Co., Ltd. (Shanghai, China). Rat polyclonal anti-NELIN (GeneTex Inc., Irvine, CA, USA; cat. no. GTX46266; 1:100 dilution), rat monoclonal anti-SM $\alpha$-actin (Novus Biologicals, Littleton, CO, USA; cat. no. NB100-74340F; 1:100 dilution), rat monoclonal anti-SRF (GeneTex Inc.; cat. no. GTX18063; 1:100 dilution), rat monoclonal anti-RhoA (Novus Biologicals; cat. no. H00000387-M03; 1:100 dilution) and rat monoclonal anti-GAPDH (cat. no. SAM1003; 1:100 dilution) antibodies were used. Horseradish peroxidase (HRP) and fluorescently-labeled sheep anti-rat IgG secondary antibody (cat. no. SE28; 1:100 dilution) was obtained from Beijing Solarbio Science \& Technology Co., Ltd. (Beijing, China). Enhanced chemiluminescence (ECL) reagent was purchased from Millipore (Billerica, MA, USA). DAPI was purchased from Beyotime Institute of Biotechnology (Jiangsu, China). Radioimmunoprecipitation assay (RIPA) lysis buffer was obtained from Sigma-Aldrich (St. Louis, MO, USA). Complete Mini was obtained from Roche Diagnostics Co. (Basel, Switzerland). A bicinchoninic acid (BCA) protein assay kit was purchased from Pierce (Thermo Fisher Scientific, Waltham, MA, USA). A nitrocellulose membrane was obtained from BioRad (Hercules, CA, USA).

Cell culture, lentiviral vector construction and infection. The present study was approved by the ethics committee of Shandong University (Jinan, China). Written informed consent was obtained from the patient, a 59-year-old male who underwent stripping of great saphenous vein. The VSMCs were cultured from a human great saphenous vein in DMEM containing $10 \%$ FBS, at $37^{\circ} \mathrm{C}$ with $5 \% \mathrm{CO}_{2}$, as described previously (13). The cells between passage 2 and 4 were then selected carefully and used. Shuttle plasmid, packaging plasmid (pGag/Pol, pRev, pVSV-G) and RNAi-Mate were mixed homogeneously for 20-25 min at room temperature. Subsequently, the transfection mixture was added into the culture dish with VSMCs drop by drop. Cell supernatant containing lentivirus particles was collected after $72 \mathrm{~h}$ of culture in an incubator with $5 \% \mathrm{CO}_{2}$ at $37^{\circ} \mathrm{C}$. Concentrated lentivirus solution was obtained from the cell supernatant following centrifugation at $100 \mathrm{x}$ g for $5 \mathrm{~min}$ and filtering using a filter (Millipore).

One day prior to infection, $1 \times 10^{5}-2 \times 10^{5}$ selected VSMCs per well were seeded in a six-well plate. The recombined lentiviral particles were diluted to a titer of $1 \times 10^{8} \mathrm{TU} / 1$ with DMEM containing 10\% FBS. The media in the six-well plate was removed and replaced with $200 \mu$ l diluted viral suspension. Following $24 \mathrm{~h}$ of incubation, the viral suspension was replaced with $2 \mathrm{ml}$ DMEM. The infected cells were selected using $2 \mu \mathrm{g} / \mathrm{ml}$ puromycin for five days, which was added $72 \mathrm{~h}$ following infection. The control (no infection, treated with medium only) and the VSMCs infected with NELIN-expression vector only were subjected to the above procedure. Cells in the NELIN-VSMC group were treated with $0.25 \%$ trypsin and diluted into single cell suspensions $\left(1.5 \times 10^{4} / \mathrm{ml}\right)$ using $2 \%$ FBS medium. These cells were then inoculated onto a 12-pore plate $(200 \mu \mathrm{l} / \mathrm{well})$ and incubated with $10 \mu \mathrm{mol} / 1 \mathrm{Y}-27632$ (Shanghai Haoran Biological Technology Co., Ltd.), a Rho kinase inhibitor, for $24 \mathrm{~h}$. All culture process were performed in an atmosphere of $5 \% \mathrm{CO}_{2}$ at $37^{\circ} \mathrm{C}$, and the experiments were performed in triplicate.

Cell morphology observation. An inverted microscope (IX70; Olympus Corporation, Tokyo, Japan) was used to observe the morphology of the control cell group, the LV-NELIN-siRNA-VSMC group and the NELIN-VSMC group.

Cytoskeleton observation by Coomassie Brilliant Blue (CBB) staining. Cells were grown on coverslips, pre-fixed using $2 \%$ paraformaldehyde for $10 \mathrm{sec}$ and then washed by phosphate buffered saline (PBS) for $1 \mathrm{~min}$. The cover slips were then dipped into $1 \%$ Triton $\mathrm{X}-100$ at $4^{\circ} \mathrm{C}$ for $30 \mathrm{~min}$ and washed with PBS for $15 \mathrm{~min}$. Subsequently, the cells were fixed with $4 \%$ paraformaldehyde for $20 \mathrm{~min}$ and stained with $0.2 \% \mathrm{CBB}$ R250 for $30 \mathrm{~min}$. Finally, the cover slips were washed by distilled water for $15 \mathrm{~min}$, dried naturally, made transparent by xylene and sealed by neutral balsam for observation under a microscope (FSX100, Olympus Corporation). 
A

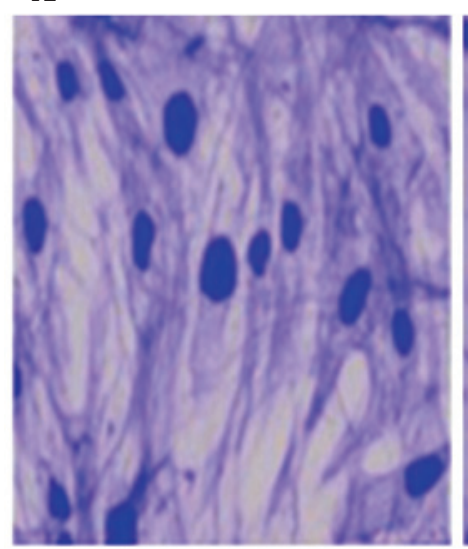

B

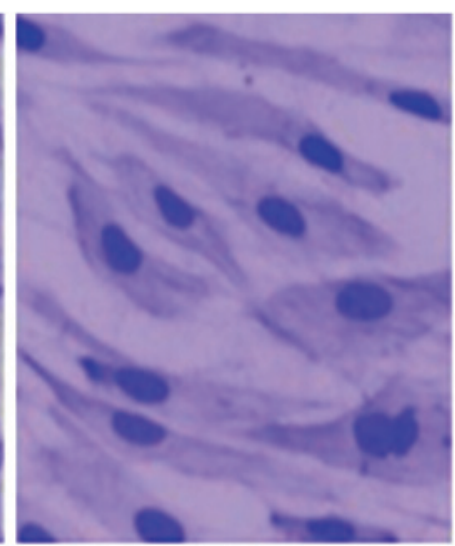

C

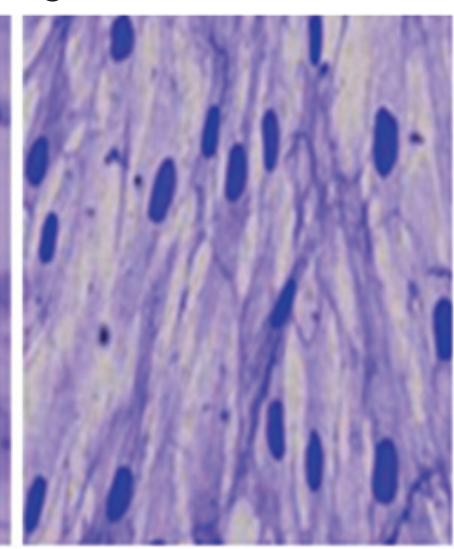

Figure 1. Morphology of VSMCs, (A) control group; (B) lentivirus-NELIN-small interfering RNA-VSMC group; (C) NELIN-VSMC group. Magnification, x200. VSMC, vascular smooth muscle cell.

Reverse transcription quantitative polymerase chain reaction $(R T-q P C R)$. VSMCs from the control group, the LV-NELIN-siRNA-VSMCs group and the NELIN-VSMCs group were selected, respectively. Total RNA was isolated from the cultured cells using TRIzol and reversely transcribed to cDNA using the PrimeScript ${ }^{\circledR} 1$ st Strand cDNA Synthesis kit according to the manufacturer's instructions. PCR was performed on an Mx3000P real-time PCR instrument (Agilent Stratagene, Amsterdam, Netherlands) using the manufacturer's instructions of the SYBR ${ }^{\circledR}$ Premix Ex Taq ${ }^{\mathrm{TM}}$ kit. The primers of target genes were designed and supplied by Invitrogen Life Technologies and shown in Table I. Each RT-qPCR reaction mix consisted of $12.5 \mu \mathrm{l} \mathrm{SYBR}{ }^{\circledR}$ Premix Ex $\mathrm{Taq}^{\mathrm{TM}}, 3 \mu \mathrm{l}$ cDNA, $0.2 \mu \mathrm{L}$ each primer $(10 \mu \mathrm{M})$ and 9.1 $\mu \mathrm{l}$ PCR-grade water, in a final volume of $25 \mu 1$. Thermal cycling conditions included an initial step at $95^{\circ} \mathrm{C}$ for $30 \mathrm{sec}$, followed by 40 cycles at $95^{\circ} \mathrm{C}$ for $5 \mathrm{sec}$ and $60^{\circ} \mathrm{C}$ for $30 \mathrm{sec}$. The final extension step included 40 cycles at $95^{\circ} \mathrm{C}$ for $1 \mathrm{~min}$, $60^{\circ} \mathrm{C}$ for $30 \mathrm{sec}$ and $95^{\circ} \mathrm{C}$ for $30 \mathrm{sec}$. Data were calculated by the ${ }^{\Delta A} \mathrm{Ct}$ method and compared to a reference (GAPDH). All experiments were performed in triplicate and repeated in at least three separate experiments.

Western blot (WB) analysis. Following lysis in cold RIPA lysis buffer containing the protease inhibitor cocktail Complete Mini, the lysate was centrifuged at 4,800 xg for $20 \mathrm{~min}$, and the supernatant was collected. Total protein was then determined using the BCA protein assay kit according to the manufacturer's instructions. Protein samples were incubated with loading buffer containing $10 \mathrm{mM}$ Tris- $\mathrm{HCl}$ ( $\mathrm{pH} 6.8), 1 \%$ (w/v) SDS, 25\% (v/v) glycerol, $0.1 \mathrm{mM}$ b-mercaptoethanol and $0.03 \%(\mathrm{w} / \mathrm{v})$ bromophenol blue. These preparations were then boiled for $5 \mathrm{~min}$ and separated using $12 \%(\mathrm{w} / \mathrm{v})$ SDS-PAGE. The protein gels were transferred onto a nitrocellulose membrane in transfer buffer (25 mM Tris, $192 \mathrm{mM}$ glycin, 20\% methanol) according to a previous method (14). Subsequently, the membrane blots were blocked with TBST containing 5\% (w/v) nonfat dry milk. The membrane was then incubated with anti-SRF, RhoA, SM $\alpha$-actin, anti-NELIN and antiserum as the primary antibody for $1 \mathrm{~h}$ at room temperature, and washed three times with TBST for $10 \mathrm{~min}$. As the secondary antibody, HRP and fluorescently-labeled antibody was used for $1 \mathrm{~h}$ at room temperature. Membranes were visualized using the ECL plus kit and exposed to Kodak X-ray film (Eastman Kodak, Rochester, NY, USA). Finally, the densitometric intensities were measured and analyzed using an LAS-3000 and Multi Gauge version 3.0 (Fujifilm, Tokyo, Japan) (15).

Immunofluorescence. The selected cells grown on a 48-well plate and fixed with $4 \%$ paraformaldehyde for $20 \mathrm{~min}$ and washed sequentially for $15 \mathrm{~min}$ with PBS. Cells were made transparent with $0.2 \%$ TritonX-100 for $30 \mathrm{~min}$ and washed with PBS for another $15 \mathrm{~min}$. The cells were then blocked with $5 \%$ goat serum for $30 \mathrm{~min}$ and incubated with SRF primary antibody $(1: 100)$ overnight at $4^{\circ} \mathrm{C}$. The incubated cells were washed with PBS for $15 \mathrm{~min}$ and subsequently incubated with the fluorescent labeled secondary antibody $\left(1: 100,37^{\circ} \mathrm{C}, 30 \mathrm{~min}\right)$. The cell nucleus was counterstained with DAPI. Following washing with PBS, the plate was sealed using glycerin. Finally, the cells were observed using confocal microscopy (FSX100; Olympus Corporation) and images were captured.

Statistical analysis. Values are expressed as the mean \pm standard error of the mean and analyzed adopting SPSS 12.0 statistical software (SPSS, Inc., Chicago, IL, USA). P $<0.05$ was considered to indicate a statistically significant difference between values. One-way analysis of variance was used for data analysis. Experiments in each group were performed thrice.

\section{Results}

Cell morphology. As shown in Fig. 1, the normal VSMCs grew well in DMEM with $10 \%$ FBS and presented a 'valley-peak' form during combination. Cells in the NELIN-VSMC group were comparatively long and thin with a cyclic cytoplasm of small volume, and grew in a luminal structure. The cytoskeleton of these cells exhibited dense grids and fasciculation as shown by CBB staining. However, cells in the LV-NELIN-siRNA-VSMC group had a larger volume and 
Table II. Real-time quantitative polymerase chain reaction of NELIN.

\begin{tabular}{lcccc}
\hline Cell group & $\mathrm{Ct}$ & $\Delta \mathrm{t}$ & $\Delta \Delta$ & $2^{-\Delta \Delta \mathrm{Ct}}$ \\
\hline Control & 28.20 & 6.06 & 0 & 1 \\
LV-NELIN-siRNA-VSMC & 29.26 & 7.77 & 1.70 & 0.31 \\
NELIN-VSMC & 24.69 & 3.48 & -2.58 & 5.98 \\
\hline
\end{tabular}

LV, lentiviral vector; siRNA, small interfering RNA; VSMC, vascular smooth muscle cells.

Table III. Real-time quantitative polymerase chain reaction of $\alpha$-smooth muscle actin.

\begin{tabular}{lcccc}
\hline Cell group & $\mathrm{Ct}$ & $\Delta \mathrm{t}$ & $\Delta \Delta$ & $2^{-\Delta \Delta}$. \\
\hline Control & 25.90 & 3.76 & 0 & 1 \\
LV-NELIN-SiRNA-VSMC & 28.32 & 6.82 & 3.07 & 0.12 \\
NELIN-VSMC & 23.59 & 2.38 & -1.38 & 2.60 \\
\hline
\end{tabular}

LV, lentiviral vector; siRNA, small interfering RNA; VSMC, vascular smooth muscle cells.

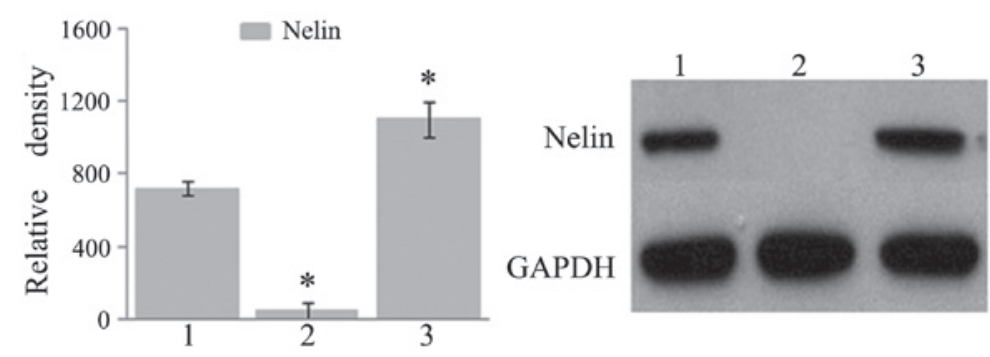

Figure 2. NELIN expression in VSMCs analyzed by western blot. 1, Control group; 2, lentivirus-NELIN-small interfering RNA-VSMC group; 3, NELIN-VSMC group. ${ }^{*} \mathrm{P}<0.05$ versus control group. VSMC, vascular smooth muscle cell.
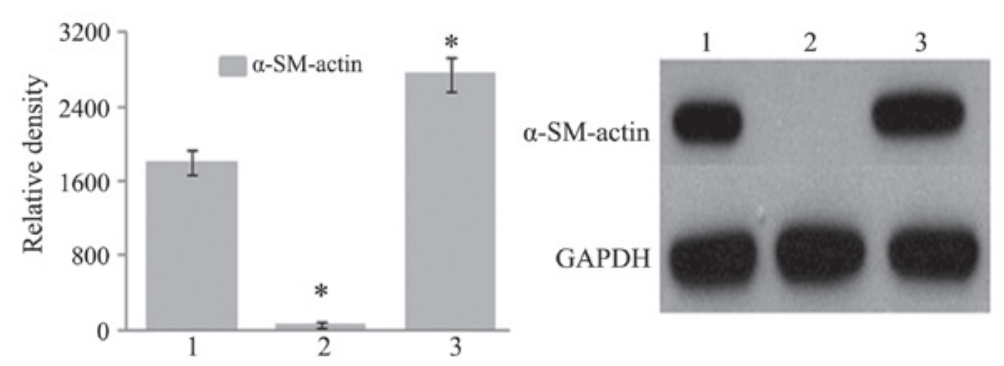

Figure 3. $\alpha$-SM-actin expression analyzed by western blot. 1, Control group; 2, lentivirus-NELIN-small interfering RNA-VSMC group; 3, NELIN-VSMC group. ${ }^{*} \mathrm{P}<0.05$ versus control group. VSMC, vascular smooth muscle cell; $\mathrm{SM}$, smooth muscle.

exhibited various shapes in the same growing environment; they had lost polarity and grew in a disordered fashion. The intensity of $\mathrm{CBB}$ staining increased, and the cytoskeleton was not clearly defined and sparsely latticed.

NELIN expression in VSMCs. Results from real-time quantitative PCR and WB showed that the expression of NELIN mRNA and NELIN protein increased in cells from the NELIN-VSMC group, while it decreased significantly in cells from the LV-NELIN-siRNA-VSMC group compared with that in the control group (Table II, Fig. 2).
SM $\alpha$-actin expression in VSMCs. Fig. 3 and Table III show the results of RT-qPCR and WB analyses of SM $\alpha$-actin. Compared with that in the control group, SM $\alpha$-actin expression in cells from the NELIN-VSMC group was significantly increased. However, in cells from the LV-NELIN-siRNA-VSMC group, the expression of $\mathrm{SM} \alpha$-actin was significantly decreased.

Effect of NELIN on RhoA and SRF expression. The WB results in Fig. 4A show that the total RhoA protein expression increased in cells from the NELIN-VSMC group and decreased in cells from the LV-NELIN-siRNA-VSMC group compared with that 
A
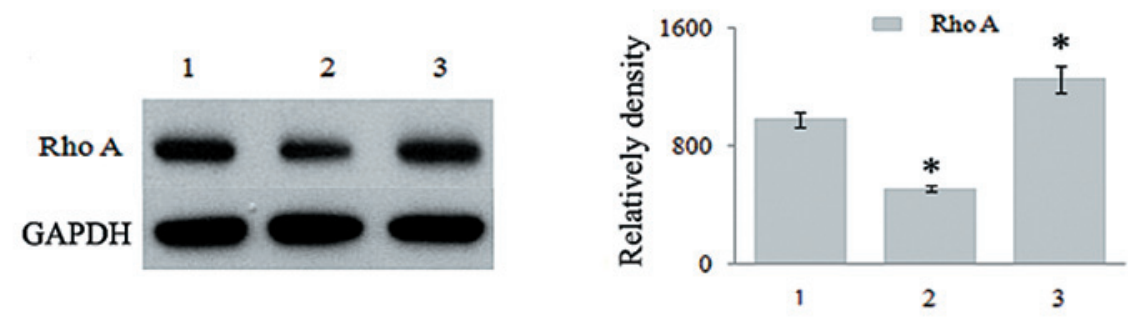

B

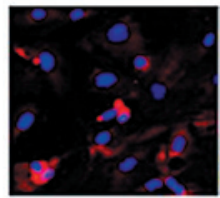

1

C

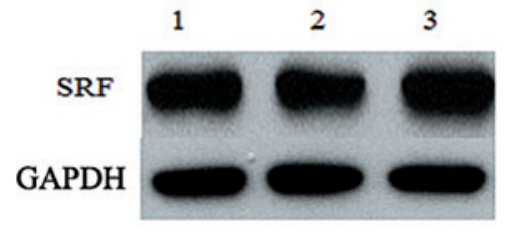

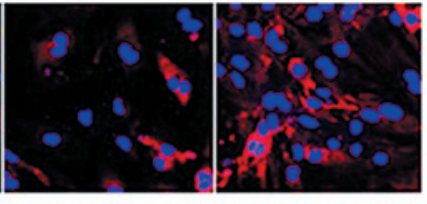

3

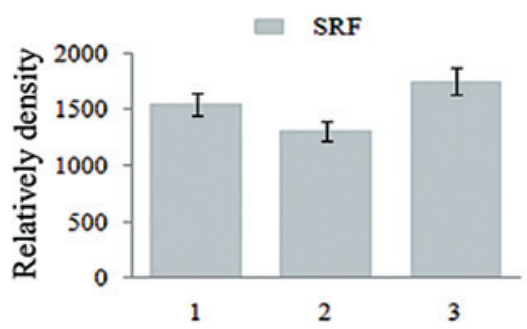

Figure 4. (A) Western blot analysis of RhoA expression in VSMCs. (B) Immunofluorescence microscopy of SRF localization and expression (red; nuclei are stained blue with DAPI) (magnification, x200). (C) Western blot analysis of SRF expression in VSMCs, 1, Control group; 2, lentivirus-NELIN-small interfering RNA-VSMC group; 3, NELIN-VSMC group. * $\mathrm{P}<0.05$ versus control group. VSMC, vascular smooth muscle cell; SRF, serum response factor; RhoA, Ras homolog gene family member A.

A

C

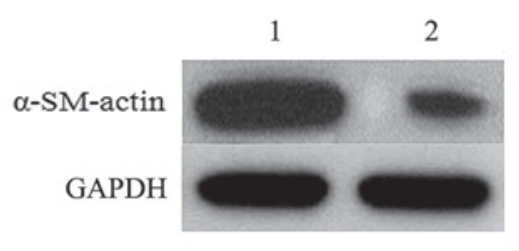

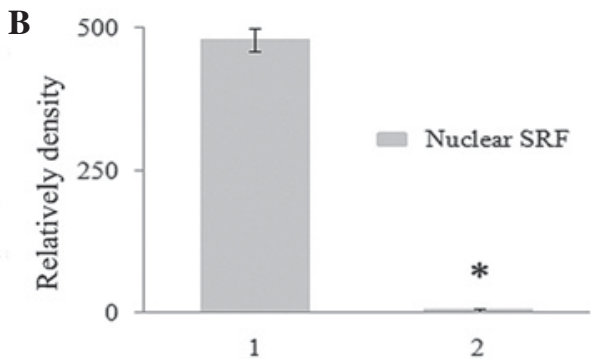

D

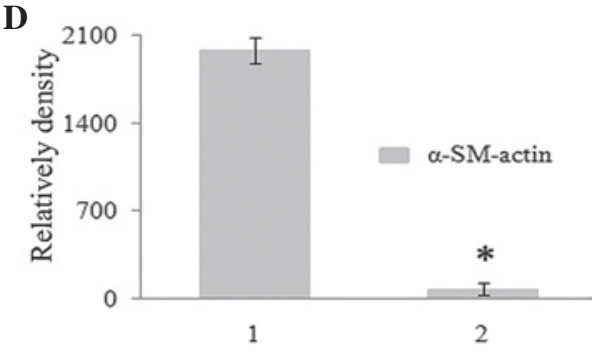

Figure 5. Western blot analysis of SRF and $\alpha$-SM-actin expression in VSMCs affected by Y-27632. (A) Western blot analysis of SRF expression in VSMCs following treatment with Y-27632. (B) Relative density of SRF expression under different conditions. (C) Western blot analysis of $\alpha$-SM-actin expression in VSMCs following treatment with Y-27632. (D) Relative density of $\alpha$-SM-actin in different groups. 1. NELIN-VSMC group 2 . Y-27632 group. "P<0.05 versus NELIN-VSMC group. VSMC, vascular smooth muscle cell; SRF, serum response factor; SM, smooth muscle.

in the control group. Immunofluorescence (Fig. 4B) showed that in the control group, SRF was predominantly expressed in the cytoplasm, while it was mainly expressed in the cell nuclei of cells in the NELIN-VSMC group, with intra-nuclear translocation. In cells of the LV-NELIN-siRNA-VSMC group, SRF was moderately expressed in the cytoplasm. WB analysis of total SRF protein showed a slight decrease of SRF levels in the LV-NELIN-siRNA-VSMC and a slight increase in the NELIN-VSMC group as compared with those in the control group; however, these differences were not statistically significant (Fig. 4C). In conclusion, the results showed that the overexpression of NELIN induced an increase of total RhoA protein expression and intra-nuclear translocation of SRF. 
Influence of Y-27632 on NELIN-induced SRF intra-nuclear translocation. Cells of the NELIN-VSMC group were incubated with $10 \mu \mathrm{mol} / 1 \mathrm{Y}-27632$, a Rho kinase inhibitor, for $24 \mathrm{~h}$, and the effect on protein expression was examined using WB analysis. The result showed that SRF and SM $\alpha$-actin expression was decreased, suggesting that the regulation of VSMC differentiation by NELIN may be mediated via the RhoA-SRF signaling pathway (Fig. 5).

\section{Discussion}

VSMCs are considered to have two phenotypes: The contractile phenotype and the synthetic phenotype (16). They are not terminally differentiated and able to modulate their phenotype in response to changing environmental cues. The phenotypic transformation process of VSMCs from the contractile to the synthetic phenotype has a critical role in the pathogenesis of varicosity, atherosclerosis and restenosis (17).

Previous studies by our group showed that NELIN expression was lower in varicose vein tissue as compared with that in normal veins $(18,19)$. Three functional domains, namely the coiled-coil region, the F-actin biding domain and the I-like domain of NELIN protein have been reported, suggesting that NELIN may be a type of F-actin binding protein (6). Furthermore, an association between NELIN expression and varicosity was found. When the synthetic phenotype of VSMCs transformed into the contractile phenotype, NELIN expression increased and the phenotypic transformation of VSMCs was stopped after NELIN expression was inhibited by NELIN-siRNA (7). This proved that NELIN was an important factor in varicose morbidity by affecting the phenotypic transformation of VSMCs. However, the mechanism underlying the phenotypic transformation of VSMCs by NELIN remained elusive. Thus, the present study explored the regulatory mechanism of NELIN in the phenotypic transformation of VSMCs.

Regulation of phenotype transformation of VSMCs by NELIN and effect on SMa-actin expression. The results of the present study showed that VSMCs with NELIN overexpression presented a contractile phenotype alongside increased $\mathrm{SM} \alpha$-actin expression as well as an increase in RhoA protein and SRF nucleoprotein expression. Following knockdown of NELIN expression, VSMCs showed a synthetic phenotype, while the expression of SM $\alpha$-actin protein, RhoA protein and SRF nucleoprotein decreased significantly. These results illustrated that the overexpression of NELIN induced SM $\alpha$-actin expression in VSMCs and promoted the transformation of VSMCs from synthetic to contractile phenotype. By contrast, VSMCs with knockdown of NELIN had the synthetic phenotype and had lost the ability to undergo positive transformation. These results indicated that NELIN may act as a key regulatory factor for the phenotypic transformation of VSMCs and may regulate $\mathrm{SM} \alpha$-actin expression.

The RhoA/SRF signaling pathway in NELIN expression regulation. Upon activation, RhoA combines with its downstream effector Rho kinase, ROCK, to further phosphorylate its downstream substrate myosin phosphatase target subunit 1 and regulate SRF-dependent smooth muscle gene expression (20). VSMC differentiation is influenced by the RhoA/Rho kinase signaling pathway via regulating SRF nuclear translocation and actin polymerization (12). SRF was reported to be able to initiate $\mathrm{SM} \alpha$-actin gene transcription by cooperating with other specific transcription factors to act on CArG, a regulatory element in the upstream promoter region of SM $\alpha$-actin (21).

The results of the present study showed an increase of RhoA protein expression and SRF intra-nuclear translocation when NELIN expression was upregulated in VSMCs, while the opposite result was observed in NELIN-knockdown VSMCs. Following incubation with the Rho kinase inhibitor Y-27632, SRF and SM $\alpha$-actin expression in the cell nucleus decreased significantly. It was demonstrated that the RhoA/SRF signaling pathway was affected by NELIN, and that phenotype transformation of VSMCs mediated by NELIN was mediated via this pathway.

The present study provided a foundation for the preventive and therapeutic application of NELIN in vascular remodeling diseases such as varicosity and atherosclerosis. However, further studies using animal experiments are required to verify the effect of NELIN in vivo.

\section{References}

1. Rzucidlo EM, Martin KA and Powell RJ: Regulation of vascular smooth muscle cell differentiation. J Vasc Surg 45, (Suppl A): A25-A32, 2007.

2. Yao QP, Zhang P, Qi YX, et al: The role of SIRT6 in the differentiation of vascular smooth muscle cells in response to cyclic strain. Int J Biochem Cell Biol 49: 98-104, 2014.

3. Zhao B, Luo X, Shi H and Ma D: Tissue factor pathway inhibitor-2 is downregulated by ox-LDL and inhibits ox-LDL induced vascular smooth muscle cells proliferation and migration. Thromb Res 128: 179-185, 2011.

4. Majesky MW: Developmental basis of vascular smooth muscle diversity. Arterioscl Throm Vas Biol 27: 1248-1258, 2007.

5. Zhao Y, Wei YJ, Cao HQ and Ding JF: Molecular cloning of NELIN, a putative human cytoskeleton regulation gene. Acta Bioch Bioph Sin 33: 19-24, 2001.

6. Wang Z, Cao HQ, Feng Y et al: Characteristics of the binding features of Nelin with F-actin and screening Nelin interactive proteins. Chin Sci Bull 49: 2487-2490, 2004.

7. PEI C, QIN S and CHEN S: Effect of Nelin on phenotypic transition of human great saphenous vein smooth muscle cells. Chin J Exp Surg 30: 925-927, 2013.

8. Xu B, Ju Y and Song G: Role of p38, ERK1/2, focal adhesion kinase, RhoA/ROCK and cytoskeleton in the adipogenesis of human mesenchymal stem cells. J Biosci Bioeng 117: 624-631, 2014.

9. Loirand G, Rolli-Derkinderen M and Pacaud P: RhoA and resistance artery remodeling. Am J Physiol Heart Circ Physiol 288: H1051-H1056, 2005.

10. Rolfe BE, Worth NF, World CJ, Campbell JH and Campbell GR: Rho and vascular disease. Atherosclerosis 183: 1-16, 2005.

11. Loirand G, Guérin P and Pacaud P: Rho kinases in cardiovascular physiology and pathophysiology. Circ Res 98: 322-334, 2006.

12. Liu HW, Halayko AJ, Fernandes DJ, et al: The RhoA/Rho kinase pathway regulates nuclear localization of serum response factor. Am J Resp Cell Mol Biol 29: 39-47, 2003.

13. Thormodsson FR and Olafsson IH: Isolation and culturing of human vascular smooth muscle cells. Methods Mol Biol 299: 197-210, 2005.

14. Towbin H, Staehelin T and Gordon J: Electrophoretic transfer of proteins from polyacrylamide gels to nitrocellulose sheets: procedure and some applications. Proc Natl Acad Sci USA 76: 4350-4354, 1979.

15. Yu HR, Kuo HC, Huang HC, et al: Glyceraldehyde-3-phosphate dehydrogenase is a reliable internal control in Western blot analysis of leukocyte subpopulations from children. Anal Biochem 413: 24-29, 2011.

16. Nagel DJ, Aizawa T, Jeon KI, et al: Role of nuclear Ca2+/calmodulin-stimulated phosphodiesterase $1 \mathrm{~A}$ in vascular smooth muscle cell growth and survival. Circ Res 98: 777-784, 2006. 
17. Hirano K: The roles of proteinase-activated receptors in the vascular physiology and pathophysiology. Arterioscl Throm Vas 27: 27-36, 2007

18. Pei C and Chen S: Detection of the nelin in peripheral blood of patients with lower extremity varicose veins and its significance. J Shandong Univ Health Sci 51: 64-66, 2013.

19. Yu X and Chen S: Expression and roles of nelin in varicose veins. Chin J Curr Adv Gen Surg 13: 1009- 9905, 2010.
20. Morelli A, Chavalmane AK, Filippi S, et al: Atorvastatin ameliorates sildenafil-induced penile erections in experimental diabetes by inhibiting diabetes-induced RhoA/Rho-kinase signaling hyperactivation. J Sex Med 6: 91-106, 2009.

21. Owens GK, Kumar MS and Wamhoff BR: Molecular regulation of vascular smooth muscle cell differentiation in development and disease. Physiol Rev 84: 767-801, 2004. 